\title{
Sarcoma-like mural nodules in cystic serous ovarian
}

\section{tumours}

\author{
T J CLARKE
}

From the Department of Histopathology, Derriford Hospital, Plymouth

SUMMARY The morphology, immunohistochemistry, ultrastructure and natural history of sarcomalike mural nodules in two serous cystic ovarian tumours are described.

Primary ovarian sarcomas are rare; they comprised only 43 of a series of 2400 ovarian tumours $(1 \cdot 8 \%){ }^{1}$ Sarcomatous and epithelial elements can be found together in ovarian tumours, most commonly in an immature teratoma or a malignant mixed mesodermal tumour (MMMT). ${ }^{1}$ Sarcomas arising in epithelial ovarian tumours are rare and those found in serous tumours are extremely rare, with only two recorded cases: a leiomyosarcoma in a serous cystadenoma, ${ }^{2}$ and a leiomyosarcoma in a serous cystadenocarcinoma. ${ }^{3}$ In this paper two further cases, diagnosed as cystic serous ovarian tumours with sarcoma-like mural, nodules are reported.

\section{Material and methods}

Sections were stained with haematoxylin and eosin, periodic acid Schiff with and without diastase pretreatment, alcian blue (pH 2.5) and Caldwell and Rannie reticulin stain. The indirect peroxidaseantiperoxidase method was used with antibodies against cytokeratin, vimentin, carcinoembryonic antigen (CEA), CA125, monoclonal antibody OC125 (from CIS UK) and myoglobin. OC125 is a monoclonal murine antibody raised against the antigen CA125 derived from the human ovarian serous papillary cystadenocarcinoma cell line OVCA $433 .{ }^{4}$ CA125 is expressed in most non-mucinous human ovarian epithelial tumours. ${ }^{56}$ Portions of the ovarian mural nodules were reprocessed from formalin fixed material and examined using a JEM-1200EX transmission electron microscope.

\section{Case reports}

CASE 1

A 49 year old, multiparous, postmenopausal woman presented with a two month history of weight loss,

Accepted for publication 25 June 1987 anorexia, and malaise. On examination she was found to have an enlarged right supraclavicular lymph node and a firm pelvic mass. At laparotomy a Tru-cut biopsy of the cervical lymph node was taken and a large, cystic left ovary and an enlarged para-aortic lymph node were removed. The left ovary was ruptured on removal but was considered to be clinically benign, and the right ovary, which appeared normal, was left in situ. The uterus and liver appeared normal and there was no ascites. A right iliac crest bone marrow trephine biopsy specimen was taken because lymphoma was suspected.

\section{CASE 2}

A 72 year old nulliparous woman presented with a three month history of anorexia, constipation, and vomiting. A fixed pelvic mass and ascites were palpable. At laparotomy ascites, peritoneal and omental metastases, and a large cystic right ovarian mass were found. A bilateral salpingo-oophorectomy, hysterectomy, and omentectomy was performed. She died eight days later. At necropsy, metastatic tumour was found in the liver, para-aortic and mediastinal lymph nodes, and encircling the small intestine in several places. The bone marrow was normal.

\section{Pathology}

\section{CASE 1}

On histological examination, the left ovary was found to be replaced by a thin walled, unilocular cyst $9 \mathrm{~cm}$ in diameter. A rough surfaced grey mural nodule measuring $3 \times 3 \times 1.5 \mathrm{~cm}$ projected into the cyst cavity. The external surface of the cyst was smooth except for slight roughening over the mural nodule.

The cyst was found to be a serous tumour of low grade ("borderline") malignancy; the columnar epithelium lining the cyst showed focal moderate cellular pleomorphism, loss of orientation, multilayering, occasional mitoses and tuft formation. The epi- 
thelium had ulcerated over the inner aspect of the mural nodule, which was covered by inflammatory exudate. The nodule was composed of sheets of large, round, and spindle cells with hyperchromatic nuclei, and there was no differentiation into recognisable connective tissue or epithelial elements. Mitoses were common and many were atypical (fig 1). The diffuse inflammatory cell infiltrate of neutrophils and eosinophils that permeated the nodule was unrelated to the occasional small areas of haemorrhage and necrosis that were present. The borders of the nodule were poorly defined, and undifferentiated neoplastic cells extended into the cyst wall adjacent to the nodule.

Reticulin stains showed a loose alveolar pattern in the mural nodule. There was no evidence of endometriosis, teratomatous organoid differentiation, osteoclast-like multinucleate giant cells, or heterologous elements. Vascular invasion by tumour cells was not seen.

CASE 2

The right ovary was replaced by a smooth surfaced, thin walled, unilocular cyst $22 \mathrm{~cm}$ in diameter with the fallopian tube stretched across it. The cyst had rup-

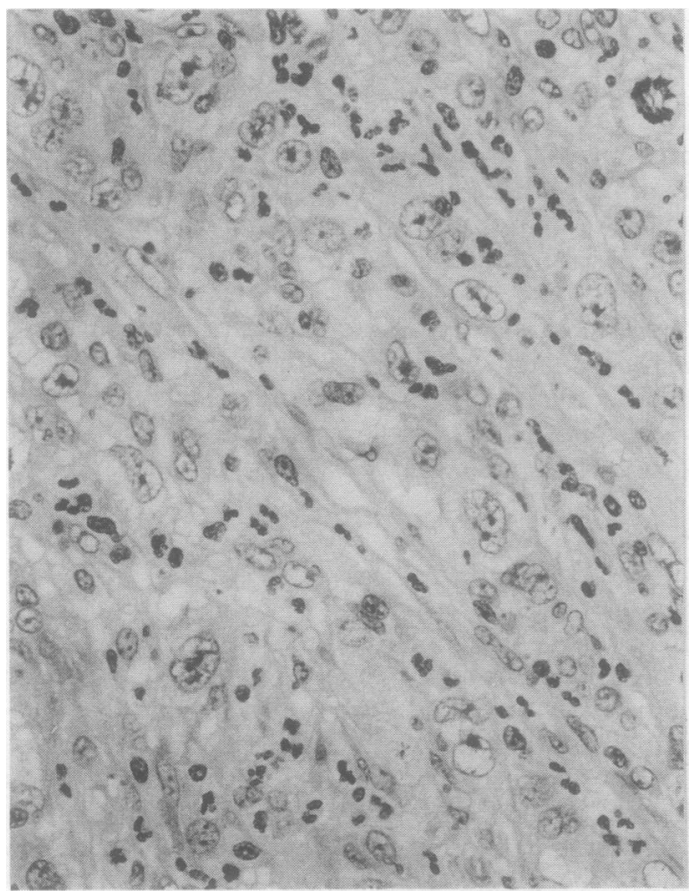

Fig 1 Case 1: detail of sarcoma-like nodule with prominent polymorphonuclear leucocyte inflammatory infiltrate. (Haematoxylin and eosin.) tured at operation. Several well circumscribed, yellowish and tan coloured mural nodules $(0.2 \mathrm{~cm}$ to $2.5 \mathrm{~cm}$ in diameter) were present on the inner surface of the cyst. A solid pale grey mass $5.5 \mathrm{~cm}$ in diameter, with a whorled cut surface, was present in the wall of the cyst. The omentum bore several grey nodules of tumour. The uterus contained a cystic endometrial polyp $2 \mathrm{~cm}$ in diameter.

The right ovarian cyst was for the most part a serous tumour of low grade ("borderline") malignancy, but there were also small foci of superficial, well differentiated invasive papillary serous adenocarcinoma containing psammoma bodies. The tan coloured mural nodules were composed of undifferentiated spindle and round cell tumour similar to that seen in case 1 , and were covered by serous epithelium (fig 2). A delicate pericellular reticulin pattern was present, in contrast to case 1 , and there was no evidence of endometriosis or teratomatous differentiation. The solid grey ovarian mass noted macroscopically was a typical ovarian fibroma. The left ovary was atrophic.

The endometrium was atrophic and bore a benign microcystic endometrial polyp with glands lined by flat, inactive epithelium. The omental deposits consis-

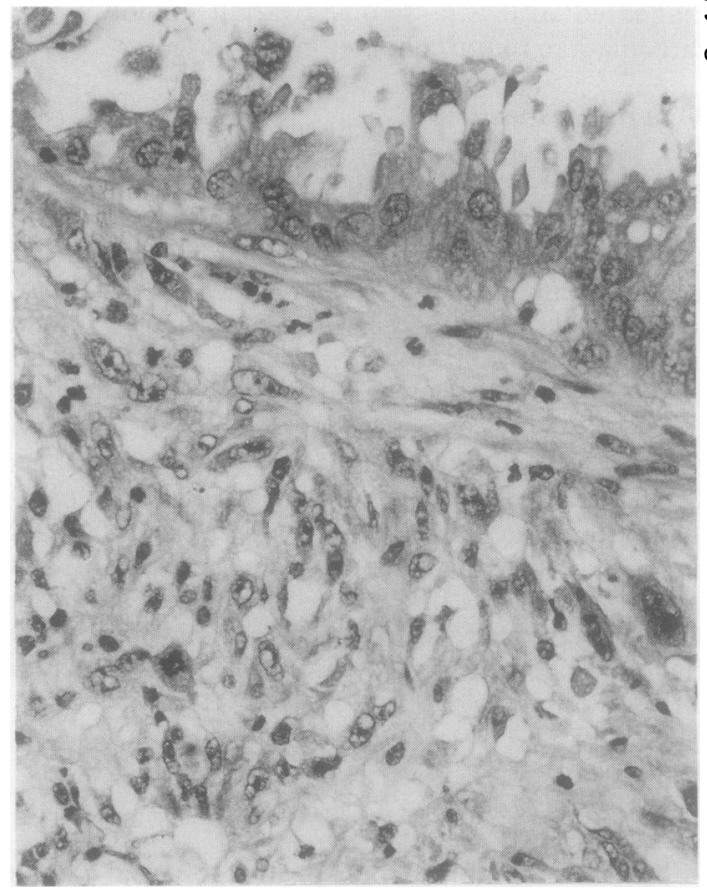

Fig 2 Case 2: sarcoma-like nodule covered by serous epithelium (Haematoxylin and eosin.) 


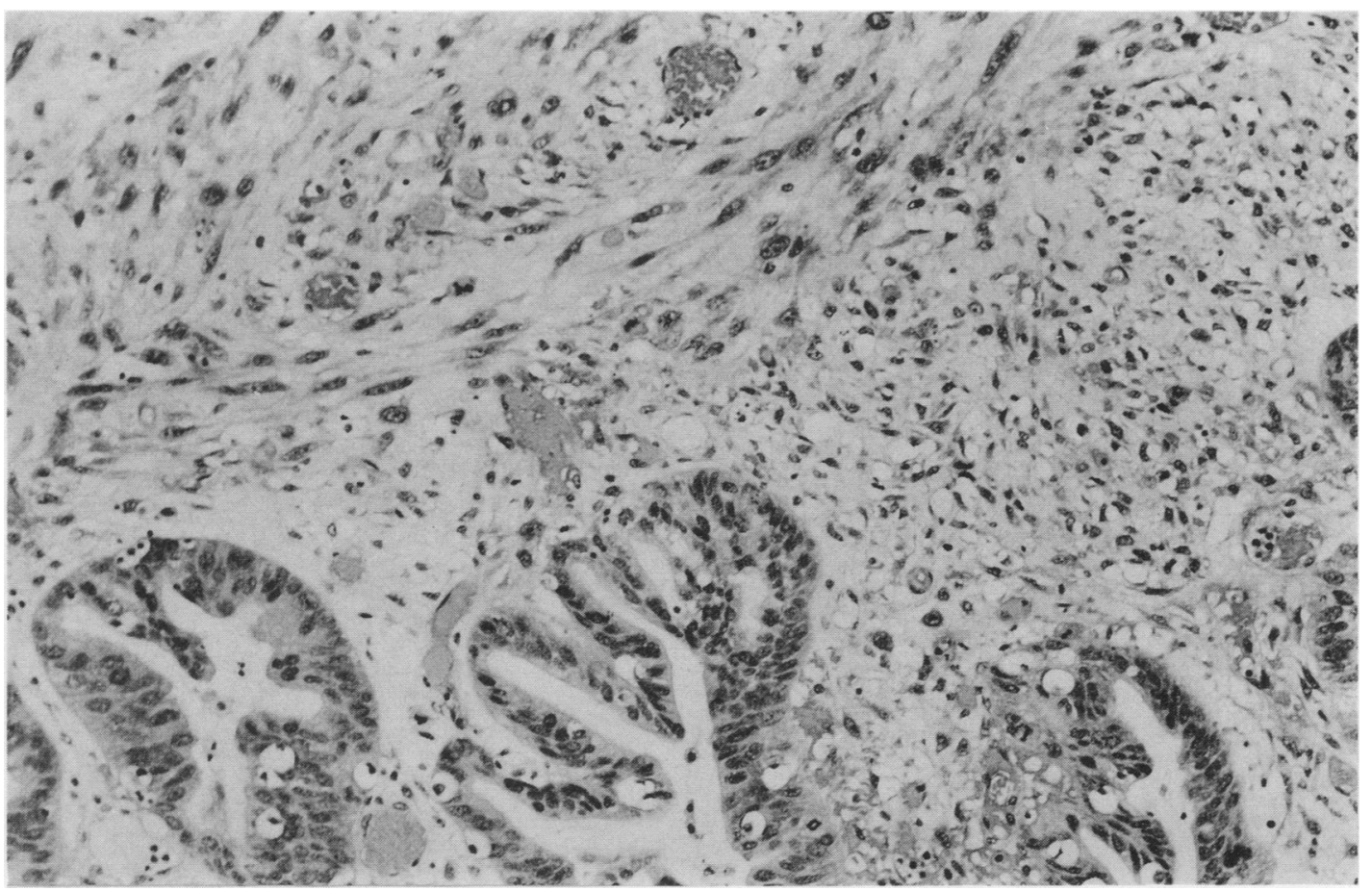

Fig 3 Case 2: omental nodule containing metastatic serous cystadenocarcinoma and spindle cell tumour. (Haematoxylin and eosin.)

ted mainly of metastatic undifferentiated spindle cell tumour similar to the ovarian mural nodules, though in some areas there was metastatic papillary serous cystadenocarcinoma (fig 3).

\section{Immunohistochemistry}

C A SE 1

The serous epithelium showed apical CA125 and cytoplasmic cytokeratin positivity and there was no CEA expression. The cells in the nodule were positive for cytokeratin and did not express vimentin, CA125, CEA, or myoglobin, nor was there intracellular neutral mucin. Ultrastructurally, the neoplastic cells contained moderate numbers of mitochondria, scanty rough endoplasmic reticulum, and occasional desmosomes with convergent tonofilaments (fig 4). Microvilli and intracellular lumina were absent and pericellular basal laminar material was scanty.

The cervical and para-aortic lymph nodes and iliac crest trephine biopsy specimen all contained metastatic undifferentiated tumour morphologically and immunohistochemically identical with that in the ovarian nodule. Despite chemotherapy with cisplatinum, adriamycin, cyclophosphamide, and numerous blood transfusions, the patient gradually deteriorated and died five months after laparotomy. An abdominal ultrasound examination shortly before

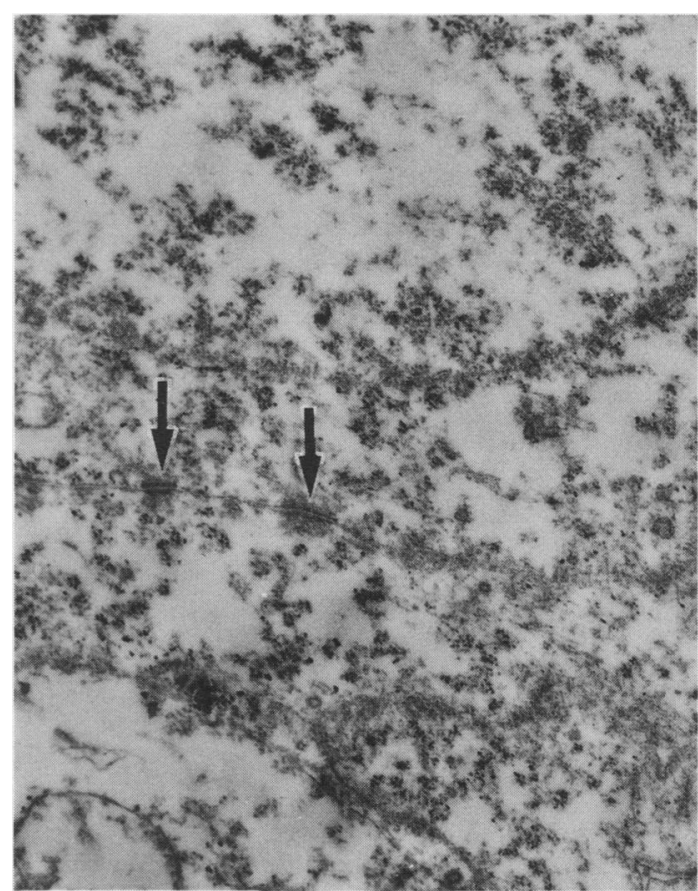

Fig 4 Case 1: ultrastructure of undifferentiated cells in nodule. Note two desmosomes (arrowed). (Uranyl acetate and lead citrate.) 


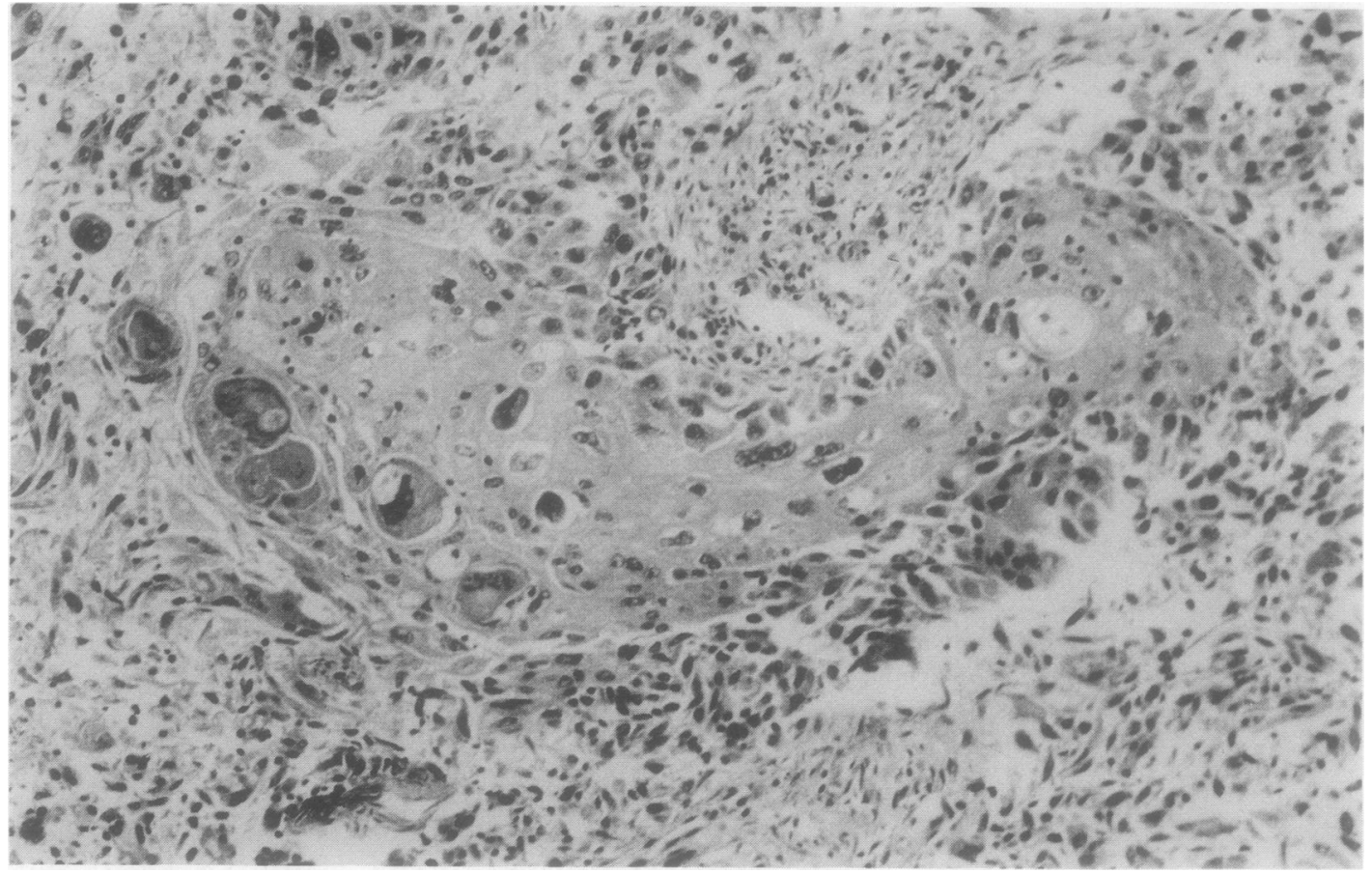

Fig 5 Case 2: metastasis adherent to small intestine containing squamous carcinoma. (Haematoxylin and eosin.)

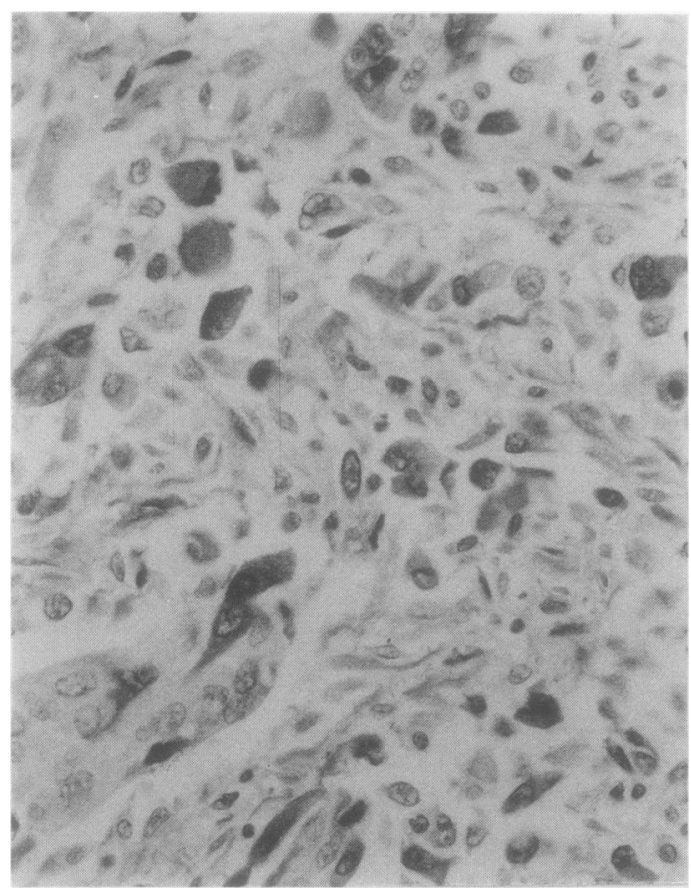

Fig 6 Case 2: hepatic metastasis containing myoglobinpositive rhabdomyoblasts. (Antimyoglobin peroxidaseantiperoxidase.) death showed hepatic metastases. Consent for necropsy was not obtained.

\section{CASE 2}

The serous lining epithelium was positive for cytoplasmic cytokeratin and apical CA125, and negative for CEA. The cells in the nodules were uniformly positive for vimentin with no expression of cytokeratin, CA125, CEA, or myoglobin. Electron microscopy of the nodules showed cells containing moderate amounts of rough endoplasmic reticulum and scanty mitochondria, surrounded by abundant basal laminar material; there was no evidence of epithelial differentiation such as microvilli, intracellular lumina, or desmosomes.

The metastases sampled at necropsy were mainly sarcomatous positive (for vimentin) with some areas of spindle cell carcinoma positive (for cytokeratin). There were also areas suggestive of squamous carcinoma (fig 5). Small numbers of tadpole shaped rhabdomyoblasts were found that had dense eosinophilic cytoplasm and no visible cross striations; these were positive for vimentin and myoglobin (fig 6).

\section{Discussion}

Sarcoma-like mural nodules have been reported in mucinous ovarian tumours, usually of low grade ("borderline") malignancy. ${ }^{7-9}$ Microscopically, they 
consist predominantly of pleomorphic spindle and oval cells exhibiting frequent and atypical mitoses. Their margins are well circumscribed and vascular, and stroma invasion is absent; variable numbers of osteoclast-like multinucleate giant cells are present. In spite of a sarcoma-like morphology the nodules show no tendency to metastasise (mean follow-up 7.5 years) and are considered to be biologically benign. ${ }^{7-9}$ They might possibly result from an unusual reaction to cyst contents $^{7}$ or to intramural haemorrhage. ${ }^{910}$ The ovarian mural nodules in the cases reported here, though they shared several morphological features with sarcoma-like nodules in mucinous cysts, were clearly malignant. The ovarian cysts in which they were found were undoubtedly of serous type and had immunohistochemical reactions characteristic of such tumours. ${ }^{1112}$

The electron microscopical structure and immunohistochemical reactions were different in the two cases. In case 1 they were those of an undifferentiated carcinoma; in case 2 they were those of an undifferentiated sarcoma. The expression of cytokeratin, absence of vimentin staining, ${ }^{13}$ and presence of desmosomes ultrastructurally in the mural nodule in case 1 were typical of an epithelial tumour. The sarcoma-like morphology persisted in the metastases in case 1 as did the expression of cytokeratin in the metastases. There was no evidence of biphasic carcinosarcomatous differentation in either the ovarian tumour or its metastases in case 1 (as was seen in case 2). It was established that the sarcoma-like mural nodule in case 1 was a primary anaplastic carcinoma arising in a serous cystadenoma of borderline malignancy. The early paraxial lymphatic spread of metastases seen in case 1 , in an apparently FIGO stage $1^{14}$ ovarian carcinoma, is characteristic of undifferentiated ovarian carcinoma. ${ }^{15}$ The malignant behaviour of the nodules in both cases was in contrast to the benign nature of reactive proliferative stromal reactions in ovarian epithelial tumours. ${ }^{16}$

In case 2 the ovarian tumour showed discrete development of sarcoma-like mural nodules projecting into a serous cystadenocarcinoma. The immunohistochemistry and ultrastructure of the sarcoma-like nodules were consistent with undifferentiated sarcoma arising from the cyst wall, in contrast to the epithelial characteristics of the sarcoma-like nodule in case 1 . The metastases in case 2 were composed of carcinomatous and sarcomatous elements with rhabdomyoblasts positive for myoglobin ${ }^{17}$ and were typical of those arising from an ovarian malignant MMMT. ${ }^{18}$ The uterus, in which MMMTs arise more commonly, was shown not to be the origin for either the ovarian mural nodules or metastases in case 2 .

The epithelial element most commonly found in ovarian MMMTs resembles papillary serous cystadenocarcinoma. ${ }^{18}$ The metastases from an ovarian MMMT are usually predominantly sarcomatous, ${ }^{18}$ and rhabdomyoblasts may be found only in the metastases. ${ }^{18}$

Although the ovarian tumour in case 2 lacked the intermingling of carcinomatous and sarcomatous elements typically found in an ovarian MMMT,18 19 it appeared to have been an early form of ovarian MMMT with separate development of carcinomatous and sarcomatous elements. The ovarian tumour and metastases in case 2 exhibited other features commonly found in ovarian MMMTs.

The histogenesis of the carcinomatous and sarcomatous elements in typical ovarian MMMTs is unknown, though an ovarian MMMT has been described in the wall of an endometriotic cyst. ${ }^{20}$ Tissue from a human ovarian MMMT, when passaged in nude mice, has shown development of discrete epithelial and mesenchymal cell lines. ${ }^{21}$ The separate developed of carcinoma and sarcoma in the ovarian tumour in case 2 reflects this finding and is the first description of a MMMT arising in an ovarian tumour of serous type that I know of.

Benign sarcoma-like nodules in serous cystic ovarian tumours have not been described. The sarcoma-like mural nodules reported here were anaplastic carcinoma and undifferentiated sarcoma (forming part of an ovarian MMMT), and differed from sarcoma-like mural nodules in ovarian mucinous tumours, both in morphology and in behaviour. This suggests that, unlike the occurrence in mucinous tumours, sarcoma-like mural nodules in a serous ovarian tumour should probably be regarded as malignant.

I thank Dr J Brindle for permission to report clinical material and Mrs J West for secretarial help.

\section{References}

1 Azoury RS, Woodruff JD. Primary ovarian sarcomas; report of 43 cases from the Emil Novak Ovarian Tumor Registry. Obstet Gynecol 1971;37:920-41.

2 von Numers C, Mikkonen R. Leiomyosarcoma arising in serous cystadenoma of ovary: report of a case. Annules Chirugiae et Gynaecologiae Fenniae 1960;49:240-4.

3 Walts AE, Lichtenstein I. Primary leiomyosarcoma associated with serous cystadenocarcinoma of the ovary. Gynecol Oncol 1977;5:81-6.

4 Bast RC Jr, Feeney M, Lazarus H, Nadler LM, Colvin RB, Knapp RC. Reactivity of a monoclonal antibody with human ovarian carcinoma. J Clin Invest 1981;68:1331-7.

5 Kabawat SE, Bast RC Jr, Bhan AK, Welch WR, Knapp RC, Colvin RB. Tissue distribution of a coelomic-epitheliumrelated antigen recognised by the monoclonal antibody $\mathrm{OC} 125$. Int J Gynecol Pathol 1983;2:275-85.

6 Koelma IA, Nap M, Rodenburg CJ, Flenren GJ. The value of 
tumour marker CA125 in surgical pathology. Histopathology 1987;11:287-94.

7 Prat J, Scully RE. Ovarian mucinous tumors with sarcoma-like mural nodules: a report of seven cases. Cancer 1979;44:1332-44.

8 Russell P, Wills EJ, Schweitzer P, Bannatyne PM. Mucinous ovarian tumors with giant cell mural nodules: a report of two cases. Diagn Gynecol Obstet 1981;3:233-49.

9 Fujii S, Konishi I, Kobayashi F, Okamura H, Yamabe H, Mori T. Sarcoma-like mural nodules combined with a microfocus of anaplastic carcinoma in mucinous ovarian tumor. Gynecol Oncol 1985;20:219-33.

10 Leschke H. Extraossare Reisenzellengeschwulste und die Deutung ihrer Entstehung in Cystadenomen des Ovar aus jungem Granulationsgewebe. Virchows Arch (Pathol Anat) 1951;320: 164-73.

11 Kabawat SE, Bast RC, Welch WR, Knapp RC, Colvin RB. Immunopathologic characterisation of a monoclonal antibody that recognises common surface antigens of human ovarian tumors of serous, endometrioid, and clear cell types. Am J Clin Pathol 1983;79:90-104.

12 Charpin C, Bhan AK, Zurawski VR, Scully RE. Carcinoembryonic antigen (CEA) and carbohydrate determinant 19-9 (CA19-9) localisation in 121 primary and metastatic ovarian tumors: an immunohistochemical study with the use of monoclonal antibodies. Int J Gynecol Pathol 1982;1:231-45.

13 Leader M, Collins M, Patel J, Henry K. Vimentin: an evaluation of its role as a tumour marker. Histopathology 1987;11:63-72.

14 International Federation of Gynaecology and Obstetrics. Classification and staging of malignant tumors of the female pelvis. Acta Obstet Gynaecol Scand 1971;50:1-7.

$15 \mathrm{Knapp} \mathrm{RC,} \mathrm{Friedman} \mathrm{EA.} \mathrm{Aortic} \mathrm{lymph} \mathrm{node} \mathrm{metastases} \mathrm{in} \mathrm{early}$ ovarian cancer. Am J Obstet Gynecol 1974;119:1013-7.

16 Ortega I, Nogales FF, Amerigo J, Fernandez-Sanz J. Carcinosarcomas and mixed mesodermal tumors of the ovary: a clinicopathologic study of six cases. Int J Gynecol Obstet 1978;15:561-5.

17 Mukai K, Varela-Duran J, Nochomovitz LE. The rhabdomyoblast in mixed mullerian tumors of the uterus and ovary. Am J Clin Pathol 1980;74:101-4.

18 Fenn ME, Abell MR. Carcinosarcoma of the ovary. Am J Obste Gynecol 1971;110:1066-74.

19 Dehner LP, Norris HJ, Taylor HB. Carcinosarcomas and mixed mesodermal tumors of the ovary. Cancer 1971;27:207-16.

20 Cooper P. Mixed mesodermal tumor and clear cell carcinoma arising in ovarian endometriosis. Cancer 1978;42:2821-31.

21 Centola GM. Long-term growth of a heterologous mixed mullerian tumor of the ovary. Invasion Metastasis 1986;6:123-32.

Requests for reprints to: Department of Histopathology, Derriford Hospital, Derriford Road, Plymouth PL68DH, England. 